\title{
MALIGNANT DISEASE IN INFANCY AND CHILDHOOD*
}

\author{
BY \\ WILFRID F. GAISFORD, M.D., F.R.C.P. \\ Professor of Child Health, University of Manchester
}

Prophylactic paediatrics, or child health, has made great advances during recent years. The considerable extensions in child health programmes, together with the advent of the sulphonamides and the antibiotics, have caused a marked decline in the infant mortality rate and in the death rate throughout childhood generally. As a result, malignant disease has assumed an increased prominence as a cause of death in children and now ranks higher than some of the commoner infectious diseases occurring in childhood.

\section{Incidence of Malignant Tumours in Childhood}

There is no evidence of any increased incidence of malignancy, except of the leukaemias which have certainly become more common. Deaths from leukaemia in children under 14 years, according to the Registrar-General's reports, were 107 in 1940, 209 in 1942, and 235 in 1945. In Manchester, Wilkinson (1948) has found a considerable increase in the past three years, the number now being seen averaging 10 per year compared with 0.7 per year for the preceding fourteen years. These figures confirm the strong clinical impression felt by most paediatricians that more cases of leukaemia are now being seen.

The following figures show the position occupied by malignant tumours (including leukaemia and Hodgkin's disease) as a cause of death in New York, U.S.A., compared with some of the common infectious fevers. They are for the three-year period 1942-44 for children under 14 years of age, and are modified from the Registry of the Memorial Cancer Centre, New York:

Tumours, 392.

Tuberculosis, 291.

Cerebrospinal meningitis, 150.

Whooping-cough, 134.

Anterior poliomyelitis, 86.

Congenital syphilis, 55.

Measles, 35.

For comparison, the position of malignant tumours as a cause of death in children of the same age-groups in England and Wales, for the five-year period 1938-42, is shown. These are compiled from the Registrar-General's reports:

* A paper read to the Section of Child Health, British Medical Association Annual Meeting, Cambridge, on Friday, July 2, 1948.
Tuberculosis, 12,052.

Whooping-cough, 6,238.

Tumours, 4,479.

Cerebrospinal meningitis, 3,879.

It is perhaps not widely enough appreciated what a large percentage of malignant tumours appear during the first five years of life; many, indeed, occur during the first three years. There is some practical significance in this fact, for at this period of life the greatest number of curable growths occur.

The American figures from the Mortality Statistics for 1939-43 and the British figures from the RegistrarGeneral's reports for 1938-42, are given in table 1 .

TABLE 1

MORTALITY FROM MALIGNANT TUMOURS IN CHILDHOOD

\begin{tabular}{c|c|c}
\hline Age-group & England and Wales & U.S.A. \\
\hline Birth-4 years & 1,942 & 5,525 \\
$5-9$ years & 1,371 & 3,150 \\
$10-14$ years & 1,116 & 2,871 \\
\hline
\end{tabular}

Although, . in the main, malignant tumours in childhood occur in sites different from those in adults, carcinoma of the stomach, rectum, uterus, and prostate, for example, being very rare, yet growths in almost every organ have been reported. Many of the childhood tumours, however, are peculiar to this age-period and are not seen in later life. The relative frequency with which the different organs or systems are affected shows a remarkable consistency, and has done so for a number of years and in a number of different countries (table 2).

In confirmation of the early age-incidence mentioned previously, it is worth noting that 50 per cent. of the cases at the Royal Manchester Children's Hospital, 60 per cent. of those at the Birmingham Children's Hospital, and 80 per cent. at the Hospital for Sick Children, Great Ormond Street, London, were under 5 years of age.

Helmholz (1931) recorded that 80 per cent. of his series of intracranial tumours and 67 per cent. of the cases with kidney tumours were also under 5 years of age. His material consisted of 750 cases seen in the paediatric department of the Mayo Clinic over the ten-year period from 1921 to 1930. 
TABLE 2

FINDINGS OF VARIOUS OBSERVERS AND AT DIFFERENT HOSPITALS SINCE DUZAN'S FIRST CONTRIBUTION TO THE SUBJECT IN 1876

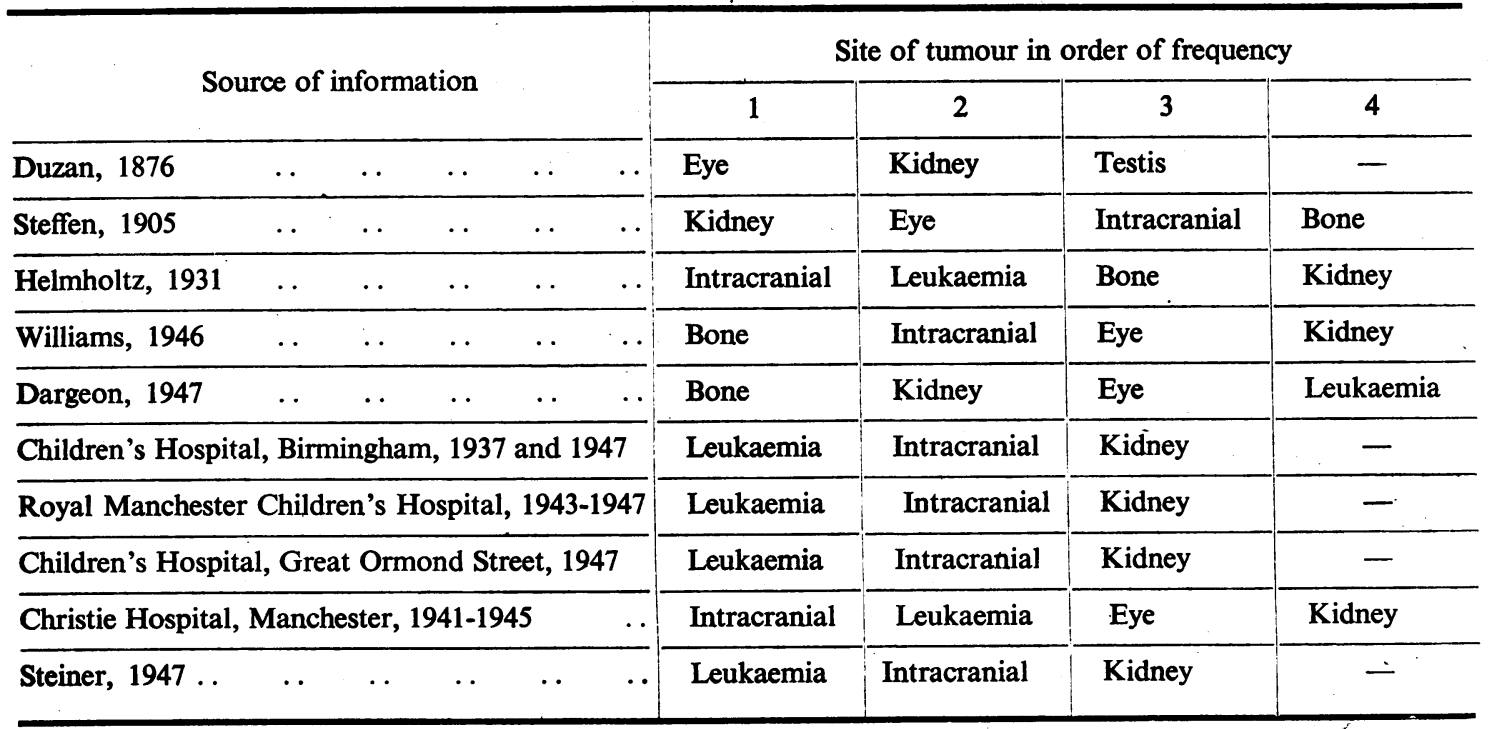

(Hodgkin's disease is included with leukaemia.)

That malignant disease in childhood generally is not a big problem numerically, may be judged from the figures shown in table 3.

As will be seen, between 0.25 per cent. and 0.5 per cent. of the admissions to a children's hospital may be expected to be malignant tumours; in a cancer institution between 1 per cent. and 2 per cent. of the total number of cases occur in children. Steiner (1947) reported that 2.49 per cent. of all deaths in the twenty-five-year period 1921-45 in the Children's Memorial Hospital, Chicago, were due to malignant disease, and that this was the cause of death in $4 \cdot 14$ per cent. of all autopsies performed at that hospital.

The foregoing figures suggest that it is unlikely that any one practitioner will see sufficient cases to keep the possibility of malignant disease constantly in the forefront of his mind when dealing with infants and children.

If it be remembered that the four commonest sites are as follows: (1) blood, (2) brain, (3) eye, and (4) kidney, and if the early signs and symptoms of these tumours are kept in mind, this would go some way towards ensuring earlier diagnosis and consequent improved prognosis as a result of the more prompt treatment which could be instituted. Particularly is this so as, with the exception of leukaemia, the tumours are capable of being cured in a great many cases.

Earlier diagnosis and treatment are, indeed, the only means of increasing the recovery rate.

\section{Diagnosis}

Dargeon (1947) has drawn attention to the two types of children who should particularly bring to mind the possibility of malignant disease: those with an unusual symptom complex and those with a silent swelling.

Intracranial neoplasms. These tend to produce changes, either constant or, more characteristically, periodic, and these become evident when a child showing such changes is compared with others of his own age or with his own previous physical stature or behaviour. In children referred to child guidance clinics because of social maladjustment or alteration in psychic or mental state, change of disposition or habits, particularly if periodic, careful search should be made to exclude a brain tumour. Helmholz (1931) recorded that 50 per cent. of his cases of intracranial tumour had had symptoms for two to six months before the correct diagnosis was reached; 25 per cent. had continued for six to twelve months; and only 10 per cent. had been diagnosed within one month of the onset of symptoms. Yet 80 per cent. of them had papilloedema and 67 per cent. had clear radiological evidence of increased intracranial pressure.

The signs and symptoms of intracranial tumour in a child differ from those in an adult because the cranial sutures do not become firmly closed until the age of about ten years. In a child with such a tumour, therefore, the head tends to enlarge progressively, Macewen's 'crackpot ' percussion sign is 
TABLE 3

MALIGNANT DISEASE IN CHILDHOOD SHOWN AS A PERCENTAGE OF TOTAL ADMISSIONS TO DIFFERENT HOSPITALS

\begin{tabular}{|c|c|c|c|}
\hline Hospital & & $\begin{array}{l}\text { Cases of malignant disease as a } \\
\text { percentage of admissions }\end{array}$ & $\begin{array}{l}\text { Cases of malignant disease in } \\
\text { childhood as percentage of total } \\
\text { admissions of malignant disease }\end{array}$ \\
\hline Children's, Birmingham .. & . $\quad \therefore$ & 0.53 (1937); 0:48 (1947) & - \\
\hline Royal Manchester Children's & .. & $0 \cdot 26(1943-47)$ & - \\
\hline Children's, Great Ormond Street & . & $0.55(1942) ; 0.6(1947)$ & - \\
\hline Children's Memorial, Chicago & . & $0 \cdot 13(1921-45)$ & - \\
\hline $\begin{array}{l}\text { Adult hospitals : } \\
\text { Christie, Manchester }\end{array}$ & . $\quad$. & - & $1 \cdot 1(1941-45)$ \\
\hline Middlesex, London & .. & - & $1 \cdot 8(1942)$ \\
\hline
\end{tabular}

present, and the skiagram shows convolution atrophy and separation of the cranial sutures.

Frontal headache is usually present but is of no localizing significance. Persistent headache, especially marked on waking, is a suggestive symptom. If there is also stiffness or tenderness of the neck, ophthalmoscopic examination should be immediately undertaken.

Ataxia is an important sign which is usually found in cerebellar tumours, and must be differentiated from the familial and other causes of cerebellar disturbance at this age.

Cyclic vomiting is another diagnosis which should not be accepted without taking the utmost care to exclude an underlying intracranial tumour. Some of Helmholz's cases had been diagnosed as cyclic vomiting, others had had appendicectomy performed because of the bouts of vomiting; many more had been treated for gastro-enteritis.

Of the many types of intracranial tumour which may occur, only two are at all common, medulloblastoma and astrocytoma. Both are subtentorial, the former usually being situated in the vermis and the latter in one of the cerebellar hemispheres. Medulloblastomas arise from embryonic rests situated just .above the fourth ventricle. In the course of growth they distend the vermis and tend to spread into the meninges and spinal cord. They are greyish-red in colour, solid and vascular. They occur more commonly in boys and are rarely seen below the age of three years. .In the last fourteen patients at the Christie Hospital, Manchester (Paterson, 1948), nine were boys and five were girls, the average age being nine years and the average duration of symptoms before treatment six months.

The first symptoms in these patients were: (1) vomiting in ten (cyclic in type in four of them),

(2) headache in nine, (3) staggering in five, and (4) pain in the back or legs in four.

These patients were all treated by irradiation; five died within nine months; nine are alive and well after more than three years.
Williams (1946) reported eighteen medulloblastomas occurring in children, the average age being eight years. There were six recoveries in the series and treatment was by irradiation. Bailey et al. (1939), treated fourteen cases surgically with one recovery.

Astrocytomas are whitish-grey in colour, firm, and containing cysts with straw-coloured coagulating contents. They give rise to symptoms similar to those caused by medulloblastomas, but usually are of longer standing. If situated in one of the hemispheres there are, in addition, homolateral inco-ordination and nystagmus. Treated surgically the results are excellent. Bailey et al. reported eighteen recoveries out of twenty-one patients treated. Comparison between their results with astrocytomas and medulloblastomas suggests that surgery is the correct treatment for astrocytomas, but not for medulloblastomas. Paterson's results point to irradiation therapy as the best treatment for medulloblastomas. The prognosis varies according to the method of treatment adopted.

Neoplasms in other sites. Asymmetrical rate of growth of one part of the body, for example, an arm or a leg, or painless swelling of the abdomen or of a testis, is suggestive of a neoplasm. The common tumour of the testis is a seminoma (carcinoma), the chief symptom of which is slow and steady enlargement of the affected testis, with occasional periodicity. Pain is not a prominent feature. In children with undescended testes metastases may provide the first symptoms: The possibility of malignancy should be considered in any scrotal swelling occurring before puberty, and if there is any doubt the chest and abdomen should be carefully examined, together with a search for any enlarged lymph glands in the left supraclavicular fossa. The inguinal glands are not affected. It is important, similarly, that in every doubtful abdominal tumour in a boy the scrotum should be examined. Treatment is by irradiation followed by 
orchidectomy, and the results are good. Williams recorded six patients of whom four were alive two and a half years or more later, and Dean found a five-year survival rate in more than three-quarters of his patients treated before metastases were evident. The Ascheim Zondek reaction is of help in diagnosing a seminoma, marked uterine enlargement occurring in the injected mouse.

Any non-traumatic swelling should be suspect, particularly a persistent adenopathy in the presence of negative Mantoux and Wassermann reactions. Any traumatic swelling which does not subside within a reasonable period of time should be similarly suspected. Progressive swelling of the abdomen in infancy, with the presence of a mass on palpation, occurs in over half the cases of Wilms's tumour, which is an embryonal adenosarcoma, resulting from a neoplastic exaggeration of the normal developmental process in the growth zone of the renal cortex in foetal life. Being encapsulated at first, this tumour causes few urinary signs in the early stages. It is a rapidly growing and vascular tumour, so that capillary rupture occurs easily and gives rise to haemorrhages within the tumour. Metastases are blood-borne, early and widespread, and the lungs and brain are particularly involved. It has been known to occur in a sevenmonth old foetus, but the average age of discovery is about three years. Boys and girls are affected almost equally, as are the two sides.

At the Christie Hospital there have been fifteen patients suffering from Wilms's tumour during the past seven years, eight boys and seven girls, and the average age was three years; eight tumours were left-sided and seven tumours right-sided. Of these fifteen patients five are alive and well after more than three years; ten patients died within two years. This tends to confirm the opinion of Ladd and White (1941) that a two-year cure is complete. They reported fourteen recoveries in sixty patients.

The first signs and symptoms noted were : (1) swelling of the abdomen in six patients, (2) haematuria in four patients, (3) abdominal pain in three patients; in two patients a mass was found in the abdomen on routine examination. The average duration of signs and symptoms before operation was four months. Five children had pre-operative irradiation in addition to the usual postoperative therapy. Of the ten patients who died, lung metastases were found in eight. It is important to remember that a palpable tumour may be the only diagnostic sign. Dean (1940) reported twenty-five patients suffering from Wilms's tumour and in no fewer than sixteen was the diagnosis made on routine examination.

Silver (1947) recorded eighteen cases, thirteen of which had nephrectomy; ten were alive from two and a quarter to fifteen years later. The youngest was an infant of three days in whom the tumour was so large that dystocia resulted. In one case pulmonary metastases appeared seven months after operation and disappeared after further irradiation, the child being alive and well two years later. In seven of the eight cases in which it was recorded, the blood pressure was raised. Nephrectomy restored it to normal, but it rose again with the appearance of metastases.

Wilms's tumour must be differentiated from the suprarenal neuroblastoma, which may present similarly as a mass in the abdomen. Metastases occur early, the liver, orbit, and skeleton being particularly involved. Like the renal embryoma (adenosarcoma), the neuroblastoma is highly malignant but is extremely radiosensitive, and Farber (1945) records recovery in ten out of forty patients. Recovery has even followed in patients in whom there had been liver metastases secondary to a primary suprarenal tumour proved histologically.

Retinal gliomas (retinoblastomas) are probably present at birth. The cells are similar to those of the partially differentiated cells of the foetal retina. These cells tend to be arranged in layers of from ten to twenty cells deep around the vessels. The cells in the peripheral layers may, therefore, die from lack of nutrition, and if the resultant necrosis becomes extensive it is possible for spontaneous cure to result.

Seventy-five per cent. of these cases are unilateral. They are not seen after the age of five years. The prognosis is good if the affected eye be enucleated before glaucoma has occurred. Williams reported twelve recoveries out of fourteen patients.

It has been suggested that if ophthalmoscopic examination were a routine part of every neonatal examination these tumours could be diagnosed at birth. As it is, 50 per cent. are diagnosable within the first two years of life, either by the presence of a dilated pupil in the affected eye, or by a white reflex, or by a squint. In bilateral cases the worse eye should be enucleated and the other one irradiated.

The early diagnosis of leukaemia, lymphosarcoma, and Hodgkin's disease (which are closely allied conditions) may be extremely difficult. Particularly is this so in leukaemia. Thrombocytopenic purpura, agranulocytosis, pertussis, acute infectious lymphocytosis, chronic non-specific infectious lymphocytosis, glandular fever, and congenital hypoplastic anaemia are some of the conditions which may have to be considered from the haematological point of view in the differential diagnosis. As not infrequently the peripheral blood picture in leukaemia may be inconclusive throughout the course of the disease, an examination of the bone marrow may be the only method of deciding with certainty. This investigation should be undertaken in any case in which doubt exists. Another common condition which may cause confusion is rheumatism, for the fever which may accompany leukaemia, together with the severe pain in the limbs and possibly also a haemic cardiac murmur, may easily lead to a mistaken diagnosis of rheumatism.

Skiagrams may be of help in diagnosing leukaemia, but not in the early stages. Caffey (1945) has drawn 
attention to their value in cases showing splenomegaly and a persistent leucopenia with pyrexia. The radiological changes are most marked at the ends of the long bones, multiple areas of destruction being visible, due to masses of leukaemic tissue causing foci of rarefaction. The periosteum may also be elevated by leukaemic penetration which results in layers of bone beyond the cortical margin.

Biopsy is usually necessary to confirm the diagnosis of Hodgkin's disease and lymphosarcoma. Both these conditions may respond dramatically to radiation therapy, and this response may be considered to be of some diagnostic significance.

Malignant bone disease tends to occur in later childhood, and so far no form of treatment has been evolved which renders the prognosis anything but grave. Ewing's tumour and osteogenic sarcoma are often first diagnosed as osteomyelitis, and nonresponse to penicillin treatment, together with subsequent radiological changes, provide the clue to the correct diagnosis. Pain is usually a prominent symptom. A history of trauma is commonly obtained, but its significance must be open to doubt in view of the strictly embryonal character of most of the tumours.

\section{The Origin of Malignant Disease in Childhood}

Heredity seems to be a minor factor, except in retinoblastoma and neurofibromatosis (von Recklinghausen's disease), which, though usually considered benign, may undergo malignant changes.

Wells (1940), in studying the records of necropsies on 3,000 newborn infants, found four undoubted malignant neoplasms, all neuroblastomas (three suprarenal and one sympathetic), and he collected sixty-six authentic cases from the literature.

It is reasonable to suppose that, in view of the early age incidence, malignant disease in childhood is usually embryonic in origin. As in ordinary circumstances a large part of the life cycle, both of humans and experimental animals, is required to produce any type of malignant tumour, some changed condition must be present to allow such early and rapid growth. Faerber (1938), has remarked on the high glycolytic metabolism of embryos (and particularly their brains and renal medullae) and on the similar glycolysis in the metabolism of tumours. This may explain the early occurrence of retinoblastomas and neuroblastomas.

Topper (1947) has recorded an interesting observation on the basal metabolism of children with tumours. She found that all cerebral tumours, benign and malignant, were associated with a low basal metabolic rate and that all other malignant tumours caused a raised basal metabolic rate. Topper explains the lowered basal metabolic rate in intracranial tumours by assuming a reduction in the amount of pituitary hormone, possibly due in part to pressure. The raised metabolic rate in other malignant tumours is assumed to be due to the vigorous metabolism of the tumour cells themselves.

\section{Characteristics of Childhood Tumours}

Certain tumours occur almost exclusively in infancy and early childhood, for example, cerebellar medulloblastoma, nasopharyngeal fibroma, malignant suprarenal neuroblastoma, Wilms's tumour, and retinoblastoma. Others are much commoner than some of those occurring in later life, such as Ewing's tumour and many of the sarcomas.

Certain other tumours are extremely rare in childhood. Carcinoma, for example, forms but a very small percentage of the malignant tumours seen at this age period. At the New York Memorial Hospital, Pack and Lefevre (1930) found that of over 16,500 patients of all ages suffering from malignant tumours, 90 per cent. were carcinoma and 10 per cent. sarcoma, whereas in the paediatric department at the Mayo Clinic, Helmholz found only 3 per cent. of carcinoma as against 97 per cent. of sarcoma amongst over 700 children. Williams, in a series of 181 children, found 8 per cent. of carcinoma and 90 per cent. of sarcoma, and Dargeon in his series of 218 malignant tumours in children found 10 per cent. of carcinoma.

Except for those that areintracranial, most tumours occurring in children are either palpable or visible, thus differing markedly from the tumours occurring in adults.

Certain embryonic tumours may cease to be malignant, either spontaneously, or after irradiation. For example, as already mentioned, retinoblastoma may undergo spontaneous cure by extensive necrosis, and suprarenal neuroblastoma may no longer show any evidence of malignancy after irradiation. Reports of such cases, confirmed by biopsy, have been published by Cushing and Wolbach (1927) and others. Farber has also noted spontaneous maturation from malignant neuroblastoma to benign ganglioneuroma. The recurring nasopharyngeal fibroma undergoes regression spontaneously at about 20 years of age. On the other hand melanomas, which are benign in childhood, may become malignant at puberty.

Histological findings and clinical progress may be at variance. Steiner has emphasized that tumours occurring in childhood often exhibit a dissociation between malignant histology and benign clinical course, and also that tumours clinically appearing to be malignant, may show no histological evidence of malignancy. For example, the recurring nasopharyngeal fibroma is histologically benign, yet it is undoubtedly a malignant growth.

There is a tendency to rapid progress and wide metastasis in childhood tumours, and although most of the growths are radiosensitive there is a liability to recurrence after treatment and careful watch must therefore be kept by periodic examinations, and further treatment instituted promptly if such recurrence becomes evident.

One important difference between tumours of children and of adults is that as children are still in process of growth this may be affected either 
lócally or generally and either excessive or diminished in rate, according to the site of the tumour, for example, pineal, pituitary, or gonadal.

\section{Treatment}

Treatment will naturally vary according to the nature of the growth and to its position and extent. It may be considered under two headings: (a) curative and (b) palliative. In the main, curative treatment will consist in irradiation or surgery or a combination of the two. If combined, irradiation may precede or follow surgery or it may be used both before and after operation. Palliative treatment will be similar, but will also include nitrogen mustard, urethane, and radioactive isotopes.

Special care must be taken in irradiation therapy; the dangers of damage to the haemopoietic system, to the epiphyses of a growing child, and to the gonads must constantly be borne in mind.

\section{Prognosis}

The prognosis varies, as might be expected, with the nature and accessibility of the tumour, the age of the child, and the length of time before treatment is started. Excluding the leukaemias, cures of practically every type of malignant neoplasm have been reported. The figures of recovery also show considerable variation. Dargeon, for example, reported eighteen cures (average duration over eight years) in his series of 218 patients with malignant disease of all types, and Williams reported sixty cures out of a series of 167 patients.

\section{Summary}

With advances in prophylactic paediatrics and the advent of the sulphonamides and antibiotics, there has been a marked difference in the last ten years in the position occupied by malignant disease as a cause of death in childhood compared with, for example; cerebrospinal meningitis, whooping-cough, measles, and diphtheria. Although there is no evidence of any increase in the total number of deaths from neoplasms, which remain rare in paediatric practice, the decline in mortality from the infectious fevers has thrown into relief the importance of malignant disease. A malignant disease may be defined as one which, untreated, leads to death of the patient either from metastasis or by direct spread.

It is still a common belief that all malignant diseases are incurable; such is no longer the case. Isolated cures have been reported in tumours of almost every form and site but, what is more important, certain types, if treated early enough, may now be said to carry an increasingly good prognosis. This is particularly true in the youngest age-groups (from birth to five years).

Although malignant disease can occur in children in any part of the body in which it appears in adults, there are certain selective sites for this disease in children, and there are scme tumours which occur in childhood only. Heredity would appear to be a factor, particularly in retinal glioma (retinoblastoma) and neurofibromatosis.

Childhood tumours grow rapidly and often metastasize widely. Because of the tendency to recurrence both locally and by metastasis, close watch must be kept after treatment in order that immediate irradiation may be given at the first evidence of such recurrence.

They differ from adult neoplasms, in that they occur during the period of active growth of the host and may, by their site, materially affect growth or metabolism.

Intracranial tumours form a considerable proportion of childhood neoplasms; their symptomatology differs considerably from that of adults.

Treatment of malignant disease may be curative, or palliative. Curative therapy consists essentially in surgery, or deep x-ray therapy, or a combination of the two. An increasing range of palliative measures is becoming available for those cases unsuitable for surgery or irradiation.

I am greatly indebted to Dr. Edith Paterson of the Christie Hospital, Manchester, for her help and advice in preparing this paper and for allowing me access to the hospital records. My thanks are also due to Professor J. M. Smellie and his registrar, and to Dr. M. Bodian for providing the Birmingham and Great Ormond Street figures quoted.

\section{REFERENCES}

Bailey, P., Buchanan, D. N., and Bucy, P. C. (1939). Intracranial Tumours in Childhood. Chicago: University of Chicago Press.

Caffey, J. (1945). Pediatric X-ray Diagnosis, p. 735. Chicago: Year Book Publishers.

Cushing, H., and Wolbach, S. B. (1927). Amer. J. Path., 2, 203.

Dargeon, H. W. (1947). Med. Clin. N. Amer., 31, 498. (1948). J. Amer. med. Ass., 136, 459.

Dean, A. L. (1940). Cancer in Childhood, ed. by H. W. Dargeon, p. 51. London: Kimpton.

Duzan, C. J. (1876). Thèse de Paris.

Faerber, E. E. (1938). Nature, Lond., 141, 1057.

Farber, S. (1946). Mitchell-Nelson's Textbook of Pediatrics. W. B. Saunders and Co., London.

Helmholz, H. F. (1931). Proc. Interstate Med. Ass. $N$. Amer., 209.

Ladd, W. E., and White, R. R. (1941). J. Amer. med. Ass., 117, 1858.

Pack, G. T., and Lefevre, R. G. (1930). J. Cancer Res., 14, 167.

Paterson, E. (1948). Personal communication.

Silver, J. (1947). J. Pediat., 30, 643.

Steffen,- A. (1905). Die malignen Geschwulste im Kindesalten. Stuttgart.

Steiner, P. E. (1947). Case Reports of Children's Memorial Hospital, Chicago, 6, 883.

Topper, Anne-(1947). Amer. J. Dis. Child., 74, 669.

Wells, H. G. (1940). Arch. Path., 30, 535.

Williams, I. G. (1946). Brit. J. Radiol., 19, 182.

Wilkinson, J. F. (1948). Personal communication. 